\title{
Proximal femoral fractures: some determinants of outcome
}

\author{
IAN F GREATOREX AND ALAN C C GIBBS \\ From the Department of Community Medicine, University of Manchester Medical School, Stopford Building, \\ Oxford Road, Manchester
}

SUMMARY We identified 226 proximal femoral fractures prospectively in people aged over 60 years during one year in Stockport (population approximately 289000 ). Our objective was to examine the association between primary treatment and outcome 6 months after the fracture using multivariate regression models. Higher haemoglobin concentrations and mental test scores on admission to hospital were associated with lower fatality within 6 months, whereas a high blood calciumphosphate product, greater age, and an active medical problem at the time of the fracture were all associated with increased fatality. An intracapsular fracture, greater age, and a greater degree of dependency before the fracture all reduced the likelihood of regaining previous walking ability by 6 months. Good walking ability before the fracture increased the likelihood of walking well by 6 months. After adjusting for the effects of these extraneous variables there was no statistically significant association between treatment and outcome.

Proximal femoral fractures are a common problem affecting elderly people and are usually treated surgically. The objective of treatment is to obtain accurate reduction and stable fixation of the fracture to enable the patient to become mobile as soon as possible; in this way it is hoped to avoid the complications of prolonged recumbency in elderly people. However, elderly people who experience proximal femoral fractures are often frail and may have co-existing medical problems which can threaten life. In one series the estimated cumulative proportion of people aged 65 years and over who survived for 6 months after the fracture was only $59.7 \% .^{1}$ It can be difficult for the surgeon to decide whether an individual patient is likely to benefit from surgery. Furthermore, in evaluating health care for proximal femoral fractures the surgeon will be interested in the effect of particular treatments on outcome.

Some authors have examined the relationship between patient variables at the time of admission to hospital and outcome. ${ }^{1-3}$ Some of these determinants of outcome may be associated with each other, for example the oldest people are more likely to be confused, and it is therefore necessary to consider their interaction when examining the association between each factor and outcome. Multivariate regression is one way of examining the association between determinants and outcome and such a model can be used to adjust for the effects of extraneous variables. In this way, the association between treatment and outcome can be isolated.

This report presents some of the findings of a prospective observational study of all proximal femoral fractures which occurred over a period of 1 year in a particular population. Outcome was assessed 6 months after the fracture in terms of survival (mortality) and walking ability (morbidity). The relationship between certain determinants measured at the time of admission to hospital and outcome was examined by modelling. The objective of the study was to examine the association between treatment and outcome after adjusting for the effects of some extraneous variables.

\section{Methods}

\section{SUBJECTS}

All new proximal femoral fractures which occurred during a 1 year period from 1 September 1984 in persons aged over 60 years, who were usually resident in Stockport, were included in a prospective observational study. The case definition included fractures proximal to and including the lesser trochanter but excluded isolated fractures of the femoral head and trochanters and subtrochanteric fractures. This definition was designed to include 
those proximal femoral fractures which cause disability in elderly people.

\section{INFORMATION}

Patients were interviewed soon after admission to hospital, in most cases before surgery. In those with evidence of confusion on a formal test of mental function, ${ }^{4}$ further information was obtained from relatives, friends or carers. Information collected on admission included personal details, medical history, details of social circumstances and an assessment of reported dependency and mobility immediately before the fracture. Dependency was assessed using an ordinal scale developed at the Benjamin Rose Hospital. ${ }^{5}$ Patients were graded on this scale according to whether they were continent of urine and faeces, were able to bath, feed and dress themselves, transfer between bed and chair and go to the toilet. They were assigned to one of eight scores on an ordinal scale of mobility: able to walk unaided (score $=1$ ), with one stick, with two sticks, with a frame, with the assistance of one person, of two people, chairbound, and bedbound (score $=8$ ). An attempt was made to visit all patients 6 months ( 182 days) after the fracture at their abode at that time. Patients or their carers were again interviewed and the gait of those patients who could walk was examined. Information about patients who had left the district was obtained by telephone interview.

\section{STATISTICAL ANALYSIS}

Independent covariates-Table 1 lists the 11 covariates which were measured soon after admission to hospital and which were examined for their association with outcome 6 months after the fracture.

\section{Table 1 Independent covariates included in the analysis}

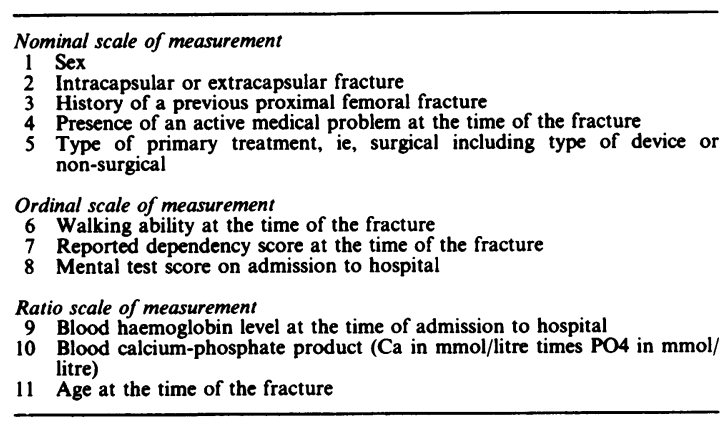

Dependent variables-Survival times to 6 months (singly censored ${ }^{6}$ ) were calculated in days after the fracture. The ordinal scores for walking ability before and 6 months after the fractures were compared and the dependent variable could take two values: 1 (walking ability at 6 months the same as or better than before the fracture-"success"), and 0 (dead, or walking ability at 6 months worse than before the fracture-"failure").

Computation-The simultaneous effects of the independent covariates on survival were analysed using Cox's proportional hazard model. ${ }^{6}$ BMDP computer program P2L was used for the analysis. ${ }^{7}$ All the variables were entered into the model and were progressively eliminated using a backward stepwise procedure. The order of removal of the variables was determined by a likelihood ratio test.

The simultaneous effects of the independent covariates on the probability of a "sucessful" outcome were examined using Cox's linear logistic regression model. ${ }^{8}$ BMDP program PLR was used and a combination of forward and backward stepping determined in which order covariates were included in the model, again using a likelihood ratio test. ${ }^{6}$

Cases with missing values for any of the covariates were excluded from the analysis.

Verbal consent to participation in the study was obtained from all patients or their relatives and the study was approved by the Stockport Ethics Committee.

\section{Results}

We identified 226 fracture cases for inclusion in the study. The mean age of the patients at the time of the fracture was 79.3 years and 193/226 patients were female. Two cases were lost to follow up at 6 months after the fracture. Further details of the subjects, the ascertainment of cases and the outcome of health care have been provided elsewhere. ${ }^{9}$

\section{SUR VIVAL}

There were missing values for at least one variable in 29 cases $(12 \cdot 8 \%)$. Five variables were included in the final regression because they were shown to have a statistically significant association with fatality within 6 months. Blood haemoglobin level and mental test score were negatively associated with fatality. A high calcium-phosphate product, advanced age and a history of an active medical problem at the time of the fracture were positively associated with fatality.

Table 2 Variables which were significantly associated with fatality within 6 months of the fracture $(n=197)$

\begin{tabular}{llllr}
\hline & & \multicolumn{2}{c}{ Relative } \\
Prognostic variable & $\chi^{2}(1 \mathrm{df})$ & p Value & risk \\
\hline Ca.PO4 product & 17.86 & 0.0001 & 17.7 \\
Medical problem & 9.14 & 0.003 & 3.1 \\
Age & 6.46 & 0.01 & 3.9 \\
Blood haemoglobin & 4.1 & 0.04 & 2.22 \\
Mental test score & 4.0 & 0.05 & 2.24 \\
\hline
\end{tabular}


Table 2 summarises the final regression. The table includes an estimate of relative risk for an individual with an arbitrary unfavourable value for any one variable with the other four variables being held at their average values. The unfavourable values for the variables were: calcium-phosphate product $5 \cdot 0$, age 90 years, a history of an active medical problem, mental test score 0 , and blood haemoglobin $8 \mathrm{~g} / \mathrm{dl}$. The relative risk was estimated with reference to an individual with a favourable value for the variable, with the other four variables being held at their average values. The favourable values for the variables were: calciumphosphate product $1 \cdot 5$, age 60 years, no active medical problem, mental test score 35 , and blood haemoglobin $14 \mathrm{~g} / \mathrm{dl}$. For example, the model predicted that an individual aged 90 years at the time of the fracture had a risk of dying during the 6 months after the fracture equal to 3.9 times that of an individual aged 60 years, the other four variables being held at their average value.

The figure is a Kaplan Meier plot which shows the observed survival of patients, stratified by the main types of primary treatment. ${ }^{10}$ Despite the observed differences in survival between the treatment groups, when the joint effects of the determinants were examined in the model there was no statistically significant association between the type of primary treatment and survival $(p=0.089)$. In other words, the model suggested that extraneous factors related to the general fitness of the patient and not the type of primary treatment best explained the observed differences in case fatality between the different treatment groups.
WALKING ABILITY

Fifty-four cases (23.9 per cent) had missing values for at least one of the variables. Table 3 summarises the final regression. For the purposes of analysis the program treated variables such as walking ability, which were measured on an ordinal scale, as if they were categorical. Age, dependency score and the presence of an intracapsular fracture all had a significant negative association with a "successful" outcome. There was a significant positive association between the patient's walking ability before the fracture and a successful outcome. Although the original scale had eight categories, there were no observations in one of these and thus the number of degrees of freedom was reduced from seven to six. There was no significant association between primary treatment and a successful outcome $(p=0.47)$.

Table 3 Covariates included in the linear logistic regression which predicted a successful outcome $(n=172)$

\begin{tabular}{lccc}
\hline Prognostic variable & $\chi^{2}$ & $d f$ & $p$ Value \\
\hline Fracture type & 4.32 & 1 & 0.0377 \\
$\begin{array}{l}\text { Age } \\
\text { Walking ability } \\
\text { before admission }\end{array}$ & 4.56 & 1 & 0.0328 \\
$\begin{array}{l}\text { Dependency score } \\
\text { on admission }\end{array}$ & 18.32 & 6 & 0.0055 \\
\hline
\end{tabular}

Table 4 summarises the goodness of fit of the model to the observed data. The model was used to predict the probability of a successful outcome for each case and the cases were classified into five groups of comparable size by this probability. The predicted

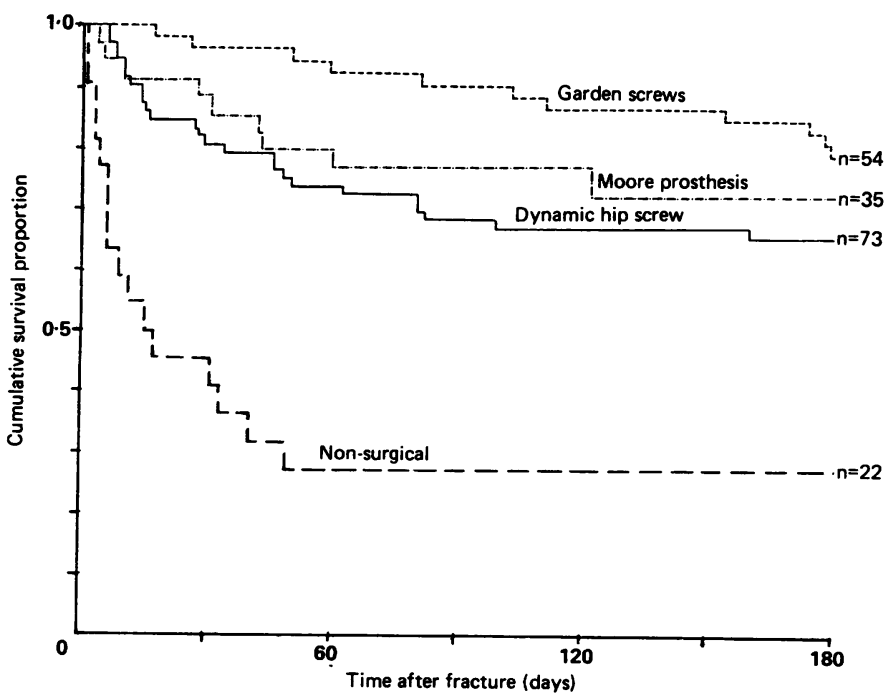

Figure Survival of patients by type of treatment. 
Table 4 Goodness of fit of the linear logistic regression $(n=172)$

\begin{tabular}{lccc}
\hline $\begin{array}{l}\text { Predicted } \\
\text { probability } \\
\text { of success }\end{array}$ & $\begin{array}{l}\text { Total number } \\
\text { of patients }\end{array}$ & $\begin{array}{l}\text { Observed number } \\
\text { of successes }\end{array}$ & $\begin{array}{l}\text { Predicted number } \\
\text { of successes* }\end{array}$ \\
\hline 0 & 34 & 0 & 0.64 \\
$0.039-$ & 36 & 3 & 2.84 \\
$0.12-$ & 33 & 6 & 5.31 \\
$0.21-$ & 33 & 11 & 8.48 \\
$0.33-$ & 36 & 15 & 17.73 \\
TOTAL & 172 & 35 & 35 \\
\hline
\end{tabular}

$\chi^{2}=1.91, \mathrm{df}=4,0.8>p>0.7$

number of successes was compared with the observed in each group. A value for $\chi^{2}$ of 1.91 with four degrees of freedom was obtained $(0.8>p>0.7)$ indicating a good fit.

\section{Discussion}

This was a prospective observational study of a particular dynamic population during 1 year. The study design was a repeat of the earlier study by Evans et al who reported the findings of a prospective survey of the outcome of all cases of proximal femoral fracture in a defined population over a period of 6 months from the time of the fracture. ${ }^{1}$ In the present study, all cases which met the restriction criteria were included, all but two were followed up at 6 months and there was no selection bias. Because the subjects were very old they were reviewed at their abode 6 months after the fracture rather than in an orthopaedic clinic. Full clinical assessment was not possible and the assessment of walking ability was inevitably somewhat subjective. Although the outcome was classified as a "success" or "failure" for the purpose of statistical analysis, this classification was not intended as a clinical judgement about the outcome in individual cases.

Although multivariate log linear regression models can identify prognostic factors with a high degree of precision in a relatively small observational series, their validity depends upon certain assumptions. For example, the simultaneous effects of the covariates are assumed to be multiplicative. There is also the problem of missing values which occur when dealing with elderly people, because of the occasional difficulty in obtaining reliable information. It is likely that those patients for whom there are missing values on any variable will differ in some way from the rest.

In the present series the type of treatment (including non-surgical treatment) was not significantly associated with outcome, whether measured in terms of fatality or morbidity. The reason for the significant association between a high calcium-phosphate product and fatality within 6 months is unexplained. This variable was included because it was felt that a low product, as would be found in osteomalacia, might be associated with poor outcome. Further analysis suggested that a high blood phosphate and not blood calcium concentration was associated with fatality. This might be because of the effect of coincidental renal impairment but this could not be examined because no other tests of renal function were performed.

The finding of the association between advanced age and poor mental function and the outcome of poor survival is consistent with other studies. ${ }^{1-3}$ Evans et al reported that advanced age, residence in an institution, and poor mental function were all negatively associated with survival up to 6 months after the fracture on multivariate analysis. ${ }^{1}$ Ions and Stevens confirmed on multivariate analysis a negative association of age and poor mental function with survival during the first six months. ${ }^{3}$

Bastow et $\mathbf{l}^{11}$ found that less well nourished elderly women with fractured neck of femur were more likely to be demented, but they concluded that the higher hospital fatality in the poorly nourished group was unlikely to be explained by the relatively small differences in mental test scores between well and poorly nourished patients. However, these authors did not examine survival to 6 months and they did not examine interactions between determinants of outcome using modelling.

The relatively poor outcome after an intracapsular fracture has also been observed previously and caused Kellog Speed to refer to the intracapsular fracture as the "unsolved" fracture. ${ }^{12}$

The present findings have implications for the evaluation of health care for elderly people in general. When adjustment was made for the effects of extraneous variables such as pre-existing morbidity and age, the type of primary treatment of the fracture had no association with either fatality or subsequent walking ability. In other words, the extraneous variables had a closer association with outcome than did the primary treatment. This suggests that a more detailed pre-operative assessment of elderly people than generally happens on acute orthopaedic wards is essential.

The fact that patients with proximal femoral fracture treated non-surgically have a poor outcome is more related to pre-existing morbidity than to the treatment itself. In any comparison of the outcome of treatment, either between different treatments or in different clinical series, it is, therefore, of the utmost importance that the patients in the different treatment groups are comparable with respect to relevant extraneous variables. The relevant variables are those which have an independent association with outcome. Since not all the relevant variables will be known, the only way of ensuring such comparability in the long run is the randomised controlled trial. Because elderly 
people commonly have multiple health problems, random allocation is particularly important when evaluating health care interventions in this client group.

We are grateful to Messrs R H L Brown, D G Wray, J A Laughton and M A Morris and to their colleagues in the North Western Region for permission to study their patients. We are also grateful to Dr Valerie Hillier and Mr Colin Ashcroft of the Department of Computation at the Manchester Medical School for assistance in the computer analysis.

Address for correspondence and reprints: Dr I F Greatorex, Specialist in Community Medicine, Salford Health Authority, Peel House, Albert Street, Eccles, Manchester M30 0NJ

\section{References}

${ }^{1}$ Evans JG, Prudham D, Wandless I. A prospective study of fractured proximal femur: incidence and outcome. Public Health 1979; 93: 235-41.

2 Young TB, Gibbs ACC. Prognostic factors for the elderly with proximal femoral fractures. Arch Emerg Med 1984; 1: 215-24.
${ }^{3}$ Ions GK, Stevens J. Prediction of survival in patients with femoral neck fractures. J Bone Joint Surg 1987; 69B: 384-7.

${ }^{4}$ Blessed G, Tomlinson BE, Roth M. The association between quantitative measures of dementia and senile changes in the cerebral gray matter of elderly subjects. $\mathrm{Br}$ J Psychiatry 1968; 114: 797-811.

5 The Staff of the Benjamin Rose Hospital. Multidisciplinary studies of illness in aged persons. II. A new classification of functional status in activities of daily living. J Chronic Dis 1959; 9: 55-62.

${ }^{6}$ Lee ET. Statistical methods for survival data analysis. Belmont, California: Life-time Learning Publications, 1980.

${ }^{7}$ Dixon WJ, Brown MB, Engleman L, et al. BMDP statistical software manual. Berkeley, Los Angeles \& London: University of California Press, 1985.

${ }^{8}$ Cox DR. The analysis of binary data. London: Methuen, 1970.

${ }^{9}$ Greatorex IF. Proximal femoral fractures: an assessment of the outcome of health care in elderly people. Community Med 1988; 10: 203-10.

${ }^{10}$ Kaplan EL, Meier P. Nonparametric estimation from incomplete observations. $J$ Am Statist Assoc 1958; 53: 457-81.

11 Bastow MD, Rawlings J, Allison SP. Undernutrition, hypothermia, and injury in elderly women with fractured femur: an injury response to altered metabolism? Lancet 1983; i: 143-6.

12 Speed K. The unsolved fracture. Surg Gynecol Obstet 1935; 60: $341-52$.

Accepted for publication July 1988 